\title{
Groupwise Combined Segmentation and Registration for Atlas Construction
}

\author{
Kanwal K. Bhatia ${ }^{1}$, Paul Aljabar ${ }^{1}$, James P. Boardman ${ }^{2}$, Latha Srinivasan ${ }^{2}$, \\ Maria Murgasova ${ }^{1}$, Serena J. Counsell ${ }^{3}$, Mary A. Rutherford ${ }^{2,3}$, Jo Hajnal ${ }^{3}$, \\ A. David Edwards ${ }^{2}$, and Daniel Rueckert ${ }^{1}$ \\ ${ }^{1}$ Visual Information Processing, Department of Computing, Imperial College London \\ ${ }^{2}$ Department of Pediatrics, Faculty of Medicine, Hammersmith Hospital \\ ${ }^{3}$ Imaging Sciences Department, MRC Clinical Sciences Centre, Hammersmith \\ Hospital, Imperial College London
}

\begin{abstract}
The creation of average anatomical atlases has been a growing area of research in recent years. It is of increased value to construct representations of, not only intensity atlases, but also their segmentation into required tissues or structures. This paper presents novel groupwise combined segmentation and registration approaches, which aim to simultaneously improve both the alignment of intensity images to their average shape, as well as the segmentations of structures in the average space. An iterative EM framework is used to build average 3D MR atlases of populations for which prior atlases do not currently exist: preterm infants at one- and two-years old. These have been used to quantify the growth of tissues occurring between these ages.
\end{abstract}

\section{Introduction}

Anatomical atlases representative of populations are of tremendous value in medical image analysis and have allowed the investigation of structural and functional characteristics of the brain. There has been a recent increase in the construction of atlases representing the average anatomy of a population [1] [2] 3] 4] 5] 6] either through using pure groupwise registration techniques or through the averaging of transformations from pairwise registrations, which are less biased towards any single subject. In addition to average structure intensity atlases, it is also valuable to have segmentations of this average shape [7]. Segmentation allows for the quanitification of structural volumes, which can be used to analyse morphological differences over time or between subjects [8] [9], or for 3D visualisation and analysis [10.

Atlases representing segmented structures are useful in determining typical anatomy in a given group. Additionally, they are often used in the segmentation of new subjects of the same population [11 [12]. The construction of corresponding intensity and segmentation atlases can be achieved either through the segmentation of images followed by the registration of these segmentations [6], or through the alignment of intensity images and the propagation of individual segmentations 11. Clearly, in the first case, the registration is dependent on the 
quality of the segmentation, and, in the second method, the final segmentation is dependent on the accuracy of the registration. Segmentation and registration would therefore appear to be complementary processes and an improvement in one is likely to lead to an improvement in the other.

Much previous work on supervised segmentation of brain MR images has used probabilisitic atlases (priors) to help classify voxels [13] [12] according to their location. However, problems may occur if the population from which the prior was created differs from that of the image to be segmented, for example, in the use of adult priors, such as the MNI305 priors, to segment child brain images [14. It may not, however, be easy to obtain representative priors, as they themseleves are created from the segmentation of multiple subjects of the same population.

There has therefore been recent development in non-rigidly aligning priors to an image to be segmented [15. The most recent work has shown that integrating the registration parameters into the Bayesian framework of maximum likelihood or maximum a-posterior estimation [16 17] benefits the segmentation. However, no attempt is made to use segmentations to assist in the registration of images. This is done in [18, where a segmented target and a floating image are registered. The transformation between these images is used to improve the segmentation in the target space. The combined segmentation, registration and modelling of sets of images is considered in [19] which iteratively registers a current estimate of the transformed intensity image to a reconstruction of the image based on its current segmentation. However, it is not obvious in their work how the integration of these methods provides an improvement over sequential techniques.

In this paper, we introduce novel groupwise combined segmentation and registration algorithms. The aim is to concurrently align to, and segment a population of images in, the average coordinate system of the population. This is defined as the coordinate system which requires least deformation from itself to all subjects in the population [4. To do this we combine methods which previously have been shown, individually, to perform well for their respective tasks - groupwise registration using the Kullback-Leibler divergence [6] and the ExpectationMaximisation (EM) algorithm for segmentation [13] [12] - and demonstrate the mutual benefit of their integration. Furthermore, we incorporate an iterative update into the segmentation process which uses the current probabilistic segmentations of the population to create representative prior models for the next iteration. The use of these dynamic models, created from the population concerned, reduces the bias as compared to priors based on standard atlases.

\section{Methods}

\subsection{The EM Algorithm for Single-Subject Segmentation}

For an image $i$, let $\mathbf{Y}$ be the collection of $J$ voxels, each with intensity $y_{j}$, i.e. $\mathbf{Y}=\left\{y_{j}, j=1,2 \ldots J\right\}$. Assume we wish to segment this image into $K$ tissue classes. Let the unknown tissue labelling $\mathbf{Z}$ be represented by a vector $\mathbf{z}$ of length $K$ for each voxel. When a voxel is classified as being tissue class $l, z_{l}=1$ and $z_{k \neq l}=0$. Each tissue class, $k$, can be assumed to approximate a Gaussian 
distribution, with mean intensity $\mu_{k}$ and variance $\sigma_{k}^{2}$, forming the distribution parameters $\theta=\left\{\mu_{k}, \sigma_{k}^{2}, k=1,2 \ldots K\right\}$. The overall image can be considered to be a mixture of Gaussian distributions, with the mixing coefficients given by the prior probabilities of each of the labels, $p\left(z_{j k}=1\right)=\pi_{j k}$, for each voxel $j$. The aim is to find the segmentation and Gaussian parameters which maximise the log-likelihood:

$$
\sum_{j} \log \sum_{k} p\left(y_{j} \mid z_{j k}=1, \theta\right) \cdot p\left(z_{j k}=1\right)=\sum_{j} \log \sum_{k} G_{j k} \cdot p\left(z_{j k}=1\right)
$$

where $G_{j k}=p\left(y_{j} \mid z_{j k}=1, \theta\right)$ gives the Gaussian probability density calculated from the distribution parameters. The EM algorithm solves this by iterating between two steps, details of the solution being given in [12]:

1. Expectation step. Calculate the posterior probability distribution, $w_{j l}=$ $p\left(z_{j l}=1 \mid y_{j}, \theta\right)$, of a voxel $j$ being labelled as tissue class $l$.

2. Maximisation step. Find the Gaussian parameters that maximise Eq. 1

\subsection{Groupwise Segmentation}

The aim of our proposed groupwise segmentation method is to segment a group of $n$ images, which have been non-rigidly aligned to their average coordinate system, using representative priors created from the current estimate of the segmentations of the individuals in the group. In order to create initial soft segmentations, $p\left(z_{i j k}=1 \mid y_{i j}, \theta\right)$, for each image, $i$, the EM algorithm is run for just a single iteration using MNI priors. The voxel-wise mean of these soft segmentations is then used to create a new prior model, $\pi_{k}^{(t+1)}$, of each class, $k$, to be used in the next iteration $t+1$ :

$$
\pi_{j k}^{(t+1)}=\frac{\sum_{i}^{n} p\left(z_{i j k}=1 \mid y_{i j}, \theta\right)}{n}=\frac{\sum_{i}^{n} w_{i j k}}{n}
$$

To avoid magnifying inaccuracies caused by bias of an initial prior model, the EM process continues with the model being updated at every iteration, using Eq. 2. However, although at each iteration the log-likelihood (and therefore the theoretical segmentation) is improved for the current model, constantly updating the model means that, overall, the log-likelihood will not converge. Instead, the algorithm is terminated when the complexity of the model converges. When the images are segmented with greatest confidence and perfectly aligned, the entropy of the model will always be at a minimum. We therefore instead aim to reduce the entropy of the model, and terminate the segmentation-registration process when this converges. The entropy of the prior model, $M$, at iteration $t$ is given by:

$$
H\left(M^{(t)}\right)=-\sum_{j} \sum_{k} \pi_{j k}^{(t)} \log \pi_{j k}^{(t)}
$$




\subsection{Groupwise Non-rigid Registration}

In order to ensure the alignment of the priors to each of the images, the images need first to be aligned to a common, and preferably average, space. This could be done using a variety of either pairwise, or groupwise, non-rigid regis-

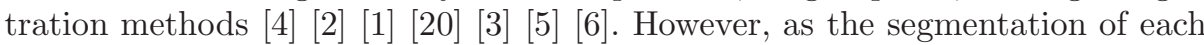
image gets more certain, this addtional knowledge can also be used to further improve the registration. The Kullback-Leibler divergence (KLD) is an additive metric for comparing distances between probability distributions. As individual segmentations become more accurate, the prior model becomes a more specific representation of the average shape. Reducing the distance between the model and each subject will therefore improve the alignment of the population to the average shape. The KLD similarity measure is given by the distance between the prior model $\pi_{j k}$ and the classification of each subject $w_{i j k}$ :

$$
\text { Minimise : } \sum_{i} \sum_{j} \sum_{k} w_{i j k} \log \left(\frac{w_{i j k}}{\pi_{j k}}\right)
$$

This aims to align each probability map with the current average probability map of the group. When they are all perfectly aligned, all distributions will be the same. This metric is particularly useful as a similarity measure for pure groupwise registration $[6$ as it reduces sensitivity to largely varying image intensities. The performance of the registration method is, however, dependent on the quality of the segmentations.

\subsection{Integrated Segmentation-Registration}

The groupwise registration method described in the previous section aims to align a group of subjects by using their segmentations. The quality of the registration is likely to be improved with improved segmentations. However, there is no requirement of the registration to provide an optimal solution to aid segmentation. The registration can instead be incorporated into a Bayesian maximum a-posteriori (MAP) framework for segmentation, as in [16]:

$$
\left(\theta^{(t+1)}, R^{(t+1)}\right)=\arg \max _{\theta, R} \log \sum_{\mathbf{Z}} P(\theta, R, \mathbf{Z} \mid \mathbf{Y})
$$

This aims to maximise the probability of the Gaussian parameters, $\theta$, the registration parameters, $R$, and the tissue labelling, $\mathbf{Z}$, given the image intensities, Y. Extending the approach in [16] to include multiple deformable images gives:

$$
\left(\theta^{(t+1)}, R^{(t+1)}\right)=\arg \max _{\theta, R} \sum_{j} \sum_{k} w_{i j k}^{(t)} \cdot\left[\log G_{j k}+\log \pi_{j k}^{(t)}\right]
$$

This can be solved using the Expectation Conditional Maximisation (ECM) algorithm 21] by optimising Eq. 6] with respect to $\theta$ and $R$ in turn. The term 
on the right-hand-side of Eq. 6 represents a cost function to be maximised. This can be rewritten by adding $\left(w_{i j k} \log w_{i j k}-w_{i j k} \log w_{i j k}=0\right)$ :

$$
\arg \max _{\theta, R} \sum_{j} \sum_{k} w_{i j k} \log \left(G_{j k} \cdot w_{i j k}\right)-w_{i j k} \log \left(\frac{w_{i j k}}{\pi_{j k}}\right)
$$

The final term represents the KLD between the posteriors and the model, and thus the alignment of the image to the group average. However, the first term depends only on the image under consideration. Maximising these two terms concurrently, as in the integrated method, need not necessarily result in improved alignment.

\subsection{Interleaved Segmentation-Registration}

An alternative method is to interleave groupwise segmentation and groupwise registration such that each term of Eq.77is optimised individually. The groupwise registration algorithm in Section 2.3 maximises only the second term of Eq. 7. Any resulting reduction in the value of the first term can be compensated for by subsequently applying the groupwise segmentation algorithm of Section 2.2. This second stage finds the Gaussian parameters that maximise only the first term without affecting the registration result. The interleaved segmentationregistration algorithm therefore aims to improve both the segmentation and the registration by iterating between the two processes.

\section{Results}

\subsection{Evaluation of Methods on Simulated Data}

A population of 100 subjects was created by deforming the MNI Brainweb image by known deformation fields (generated using a free-form deformation model based on B-splines 22]), which were constrained to sum to zero deformation across the population. The MNI Brainweb image also has ground truth and probabilistic segmentations for WM, GM, CSF and background (BG) tissue classes. The transformations to the average space, as well as the segmentations of the individual images are therefore known. In addition, varying levels of Gaussian noise (with zero mean and standard deviation ranging from 0-5) were applied across the population. The proposed methods of interleaved (INLVD) and integrated (INTGD) segmentation-registration approaches have been tested on the simulated dataset. An initial estimate of the alignment is used, created using a groupwise registration approach based on B-splines [4] with a KLD similarity metric. The MNI305 priors are used as the initial prior probability maps. Additionally, the groupwise segmentation method alone has been applied, using the known, actual transformations to average space (GW).

Effect on segmentation. The segmentation of each subject using the described techniques is compared to that obtained using the standard EM algorithm on 
each individual, using affinely-aligned MNI priors. Average Dice overlaps between the known and obtained segmentations are computed for each structure and are shown in Table 1 It can be seen that the use of groupwise segmentationregistration improves the accuracy and consistency of the segmentation.

Table 1. Mean and standard deviation of dice similarity for each structure using each segmentation method

\begin{tabular}{|c||c|c|c|c|}
\hline Method & BG & CSF & GM & WM \\
\hline \hline EM & $0.976 \pm 0.0044$ & $0.779 \pm 0.0247$ & $0.871 \pm 0.0067$ & $0.922 \pm 0.0066$ \\
\hline INGTD & $0.988 \pm 0.0009$ & $0.847 \pm 0.0063$ & $0.882 \pm 0.0037$ & $0.949 \pm 0.0023$ \\
\hline INTLV & $0.988 \pm 0.0009$ & $0.850 \pm 0.0060$ & $0.884 \pm 0.0038$ & $0.950 \pm 0.0023$ \\
\hline GW & $0.989 \pm 0.0008$ & $0.862 \pm 0.0059$ & $0.891 \pm 0.0036$ & $0.955 \pm 0.0021$ \\
\hline
\end{tabular}

Effect on registration. Both integrated and interleaved methods used constrained groupwise registration [4, using the KLD similarity measure, to ensure the atlas represents average shape of the population. The average root-meansquared (RMS) error of the final voxel deformations from the original shape were calculated. The average RMS deformation of the unregistered population was $2.92 \mathrm{~mm}$. The average error after only groupwise registration was found to be $1.93 \mathrm{~mm}$. This improved to $1.70 \mathrm{~mm}$ when using the interleaved method of segmentation-registration. However, as predicted, no improvement was found from the integrated method which resulted in a final RMS error of $1.97 \mathrm{~mm}$.

\subsection{Average Brain Atlases of Preterm Infants at One and Two Years}

Populations of 22 preterm-born subjects imaged at one year and again at two years were initially aligned to their average shapes, by averaging pairwise transformations [2]. The pure groupwise segmentation algorithm was run on each of these aligned populations, using the MNI305 priors for the initial iteration. The prior atlases were recalculated after each iteration. Convergence of these atlases for the two-year-old population is shown in Fig. 11 Fig. 2 shows the final average intensity and tissue class probability atlases for the average shape of each population.

The model of priors has additionally been used to calculate volumes of the segmented tissue classes of each average shape. It was found that the mean volume of WM increases from 278 to $326 \mathrm{~cm}^{3}(+17 \%)$ and the mean volume of GM increases from 536 to $612 \mathrm{~cm}^{3}(+14 \%)$ between one and two years.

\section{Discussion}

The groupwise segmentation-registration methods developed have been used to create average intensity and probabilistic segmentations of populations, representing their average shape. Additionally, these techniques have been used to 


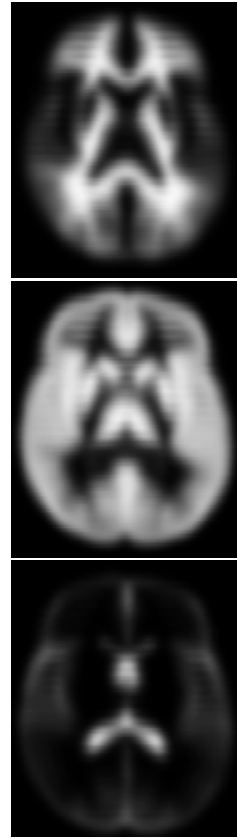

(a)
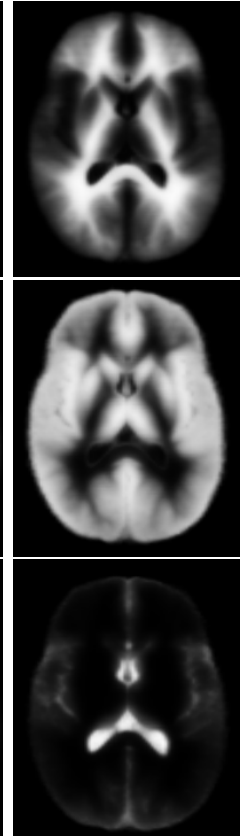

(b)
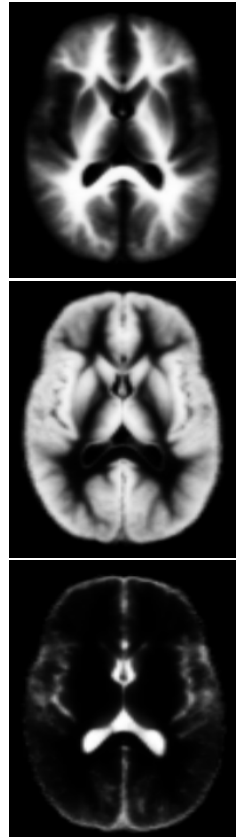

(c)
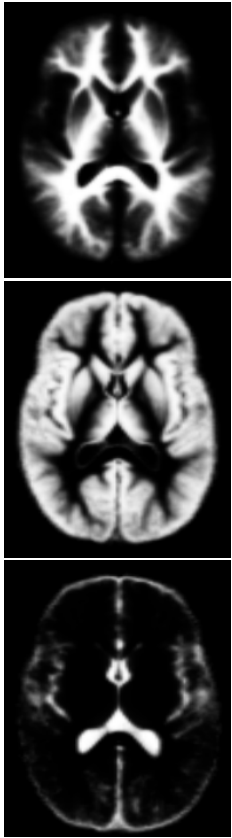

(d)
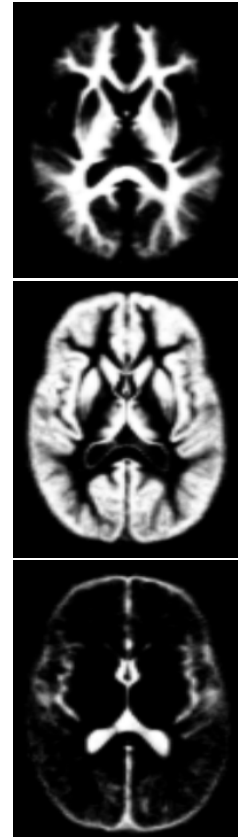

(e)

Fig. 1. The evolution of prior models for the two-year-old population for WM (top row), GM (middle row) and CSF (bottom row). (a) MNI 305 priors; (b)-(d) updated models at iterations 1-3; (e) convergence of model entropy at iteration 6 .
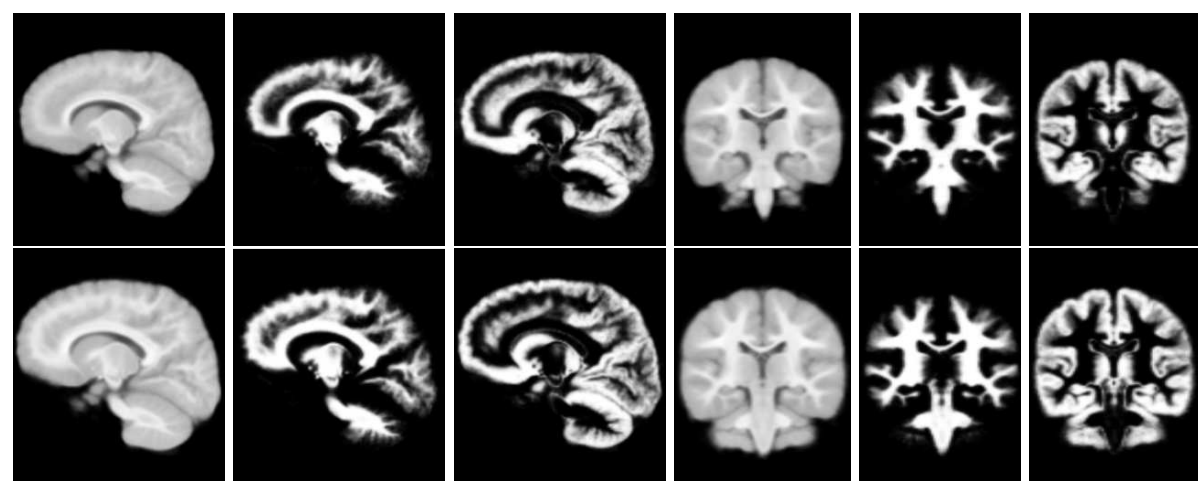

Fig. 2. Average shape atlases of 22 one- (top row) and two-year-olds (bottom row). L-R: sagittal intensity, WM and GM atlases, coronal intensity, WM and GM atlases. 
segment the individual subjects of each population in their native space. On the simulated dataset, both combined segmentation-registration methods outperform the EM algorithm at segmentation of the original images. The best segmentation occurs when the images are perfectly aligned, as obtained when the known transformations to the average space are applied. However, only the interleaved method, which explicitly aims to maximise the registration, improves the alignment of the images over that obtained by pure groupwise registration.

The groupwise segmentation algorithm has also been used to create average shape and probabilistic segmentation atlases of populations of preterm infants at one- and two-years-old, as well as hard segmentations of the individual subjects in their native space. The atlases obtained have been used to quantify tissue growth between one and two years.

It is acknowledged that validation on a simulated dataset is limited as real populations contain differing brain topologies and imaging artifacts such as noise and inhomogeneity. Manual segmentations of the infant dataset are not available at time of writing, and it is intended to compare the results on real data to gold standard segmentations in the future.

\section{References}

1. Guimond, A., et al.: Average brain models: A convergence study. Computer Vision and Image Understanding 77(9), 192-210 (2000)

2. Rueckert, D., et al.: Automatic construction of 3-d statistical deformation models of the brain using nonrigid registration. IEEE TMI 22(8), 1014-1025 (2003)

3. Joshi, S., Davis, B., Jomier, M., Gerig, G.: Unbiased diffeomorphic atlas construction for computational anatomy. NeuroImage 23, S151-S160 (2004)

4. Bhatia, K.K., et al.: Consistent groupwise non-rigid registration for atlas construction. In: ISBI 2004, pp. 908-911 (2004)

5. Christensen, G.E., Johnson, H.J., Vannier, M.W.: Synthesizing average 3D anatomical shapes. NeuroImage 32(1), 146-158 (2006)

6. Lorenzen, P., et al.: Multi-modal image set registration and atlas formation. Medical Image Analysis 10(3), 440-451 (2006)

7. Xu, S., et al.: Group mean differences of voxel and surface objects via nonlinear averaging. In: ISBI 2004, pp. 758-761 (2006)

8. Aljabar, P., et al.: Analysis of growth in the developing brain using non-rigid registration. In: ISBI 2004, pp. 201-204 (2006)

9. Boardman, J.P., et al.: Abnormal deep grey matter development following preterm birth detected using deformation-based morphometry. NeuroImage 32 (2006)

10. Kikinis, R., et al.: A digital brain atlas for surgical planning, model-driven segmentation, and teaching. NeuroImage 2, 232-241 (1996)

11. Hammers, A., et al.: Three-dimensional maximum probability atlas of the human brain, with particular reference to the temporal lobe. HBM 19, 224-247 (2003)

12. van Leemput, K., et al.: Automated model-based bias field correction of MR images of the brain. IEEE TMI 18(10), 885-896 (1999)

13. Wells, W.M., et al.: Adaptive segmentation of MRI data. IEEE TMI 15(4), 429-442 (1996)

14. Murgasova, M., et al.: Segmentation of brain MRI in young children. In: Larsen, R., Nielsen, M., Sporring, J. (eds.) MICCAI 2006. LNCS, vol. 4190, pp. 687-694. Springer, Heidelberg (2006) 
15. D'Agostino, E., et al.: Non-rigid atlas-to-image registration by minimization of class-conditional image entropy. In: Barillot, C., Haynor, D.R., Hellier, P. (eds.) MICCAI 2004. LNCS, vol. 3216, pp. 745-753. Springer, Heidelberg (2004)

16. Pohl, K., et al.: A Bayesian model for joint segmentation and registration. NeuroImage 31, 228-239 (2006)

17. Ashburner, J., Friston, K.: Unified segmentation. NeuroImage 26, 839-851 (2005)

18. Chen, X., et al.: Simultaneous segmentation and registration of medical image. In: Barillot, C., Haynor, D.R., Hellier, P. (eds.) MICCAI 2004. LNCS, vol. 3216, pp. 663-670. Springer, Heidelberg (2004)

19. Petrovic, V., et al.: Automatic framework for medical image registration, segmentation and modeling. In: MIUA 2006 (2006)

20. Twining, C., et al.: A a unified information-theoretic approach to groupwise nonrigid registration and model building. In: Christensen, G.E., Sonka, M. (eds.) IPMI 2005. LNCS, vol. 3565, pp. 1-14. Springer, Heidelberg (2005)

21. Meng, X.L., Rubin, D.B.: Maximum likelihood estimation via the ECM algorithm: A general framework. Biometrika 80(2), 267-278 (1993)

22. Rueckert, D., et al.: Non-rigid registration using free-form deformations: Application to breast MR images. IEEE TMI 18(8), 712-721 (1999) 